\title{
Daños en casas de madera ocasionados por coleópteros xilófagos, IX Región, Chile
}

\author{
Damages to wooden houses caused by xylophagus Coleoptera, \\ IX Region, Chile
}

\author{
Ramón Rebolledo R. ${ }^{*}$, Patricio Sáez B. ${ }^{1}$, Carlos Klein K. ${ }^{1}$, Cristián Salas E. ${ }^{1}$, Alfonso Aguilera P.., 2 \\ * Autor de correspondencia: ${ }^{1}$ Universidad de La Frontera, Facultad de Ciencias Agropecuarias y Forestales, \\ Casilla 54-D, Temuco, Chile, ramonr@ufro.cl. ${ }^{2}$ INIA Carillanca, Casilla 58-D, Temuco, Chile
}

\begin{abstract}
SUMMARY
In Padre Las Casas in the IX Region of La Araucanía, wood buildings have serious deterioration problems caused by xylophages Coleopteran producing structural and aesthetic damage. In this survey, subterranean termites were not found. In four different sectors of the city, the damage was evaluated in 98 houses randomly sampled, in which the damage was quantified and the relationship damage-age was established. The total built surface, the presence or absence of damage, type of wood damaged, degree of elaboration and level of deterioration were estimated. Wooden parts with symptoms of damage caused by adult insects were collected for later laboratory studies and for species identification. A total of 71 houses presented damage ( $70 \%$ of the total), being the sector Villa los Canelos the most affected, with 89\%. Structural exotic wood presented the highest damage with 54\%, followed by its combination with native ones with $14 \%$; native wood showed only $2 \%$ of damage. Painted wood presented $44 \%$ of damage, due mainly to its age in most houses. The species associated to wood in service were the common furniture beetle Anobium punctatum Hadrobregmus punctatipennis and the wood-boring weevil Pentarthrum huttoni.
\end{abstract}

Key words: Anobium punctatum, Hadrobregmus punctatipennis, Pentarthrum huttoni, Padre Las Casas.

\section{RESUMEN}

En el sector urbano de la comuna de Padre Las Casas, IX Región de La Araucanía, las viviendas tienen serios problemas de deterioro ocasionado por insectos xilófagos del orden Coleoptera que producen daños estructurales y estéticos, no detectándose, en esta ocasión, la presencia de la termita subterránea. En cuatro sectores de la ciudad se muestrearon 98 viviendas al azar, en las cuales se cuantificó el daño y su relación con el año de construcción. Se estimó también la superficie total construida, la presencia o ausencia de daño, tipo de madera atacada, grado de elaboración de la misma y niveles de deterioro. En laboratorio se analizaron trozos de madera con síntomas de daño y se obtuvieron ejemplares de insectos adultos para la determinación de las especies. Los resultados indican que el $70 \%$ de las viviendas presentaron síntomas de deterioro. La madera de construcción que presentó mayor daño correspondió a la madera exótica con un 54\%, seguida de la combinación de éstas con las nativas con un $14 \%$ y las de madera nativa con sólo un $2 \%$. Las especies de coleópteros encontrados, asociados con madera en servicio, correspondieron a los anóbidos Anobium punctatum y Hadrobregmus punctatipennis y al curculiónido Pentarthrum huttoni.

Palabras clave: Anobium punctatum, Hadrobregmus punctatipennis, Pentarthrum huttoni, Padre Las Casas.

\section{INTRODUCCIÓN}

El sector urbano de la comuna de Padre Las Casas ha sufrido, en las últimas décadas, un importante deterioro causado por insectos xilófagos que han provocado alarma en la población debido a que los daños se atribuyen, erróneamente, a la termita subterránea. Estudios preliminares en terreno realizados por los autores del presente trabajo concluyeron que los agentes causales correspon- dían a coleópteros xilófagos pertenecientes a las familias Anobiidae y Curculionidae. La presente investigación surgió como necesidad de determinar las especies involucradas y la evaluación de los daños en madera en servicio.

A nivel mundial, los trabajos de mayor interés sobre especies de coleópteros asociados a madera en servicio corresponden a Torres (1968), Coulson y Writter (1990), Creffield (1996), Yela (1997) y Dajoz (2001). En térmi- 
nos generales, los trabajos publicados sobre coleópteros xilófagos asociados a madera en servicio en Chile son escasos y en los últimos diez años cabe destacar las contribuciones de Prado (1991), Barriga et al. (1993), Artigas (1994), Apablaza (2000), Klein y Waterhouse (2000), quienes entregan antecedentes bibliográficos sobre las especies en cuestión y su distribución a nivel nacional, además de algunos aportes sobre magnitud de daño. Trabajos más recientes y actualizados se encuentran en Vargas (2002), Rojas y Gallardo (2004) y Ripa y Luppichini (2004).

\section{MÉTODOS}

El presente trabajo se llevó a cabo en el sector urbano de la comuna de Padre Las Casas, ubicada en el llano central de la IX Región de La Araucanía, entre los paralelos $38^{\circ} 46^{\prime}$ latitud sur y los meridianos $72^{\circ} 38^{\prime}$ longitud oeste (IGM., 1993) y se originó como resultado de la prospección para determinar la eventual presencia de la termita subterránea como causante del daño en casas de madera de la ciudad. El muestreo se realizó desde noviembre de 2001 a marzo de 2002, utilizando como material de estudio casas de madera, ubicadas en los sectores identificados por el Municipio de Padre Las Casas con problemas de deterioro estructural. En dicho Municipio se obtuvo la información necesaria sobre el número de viviendas de los sectores muestreados. Los valores entregados fueron estimados a partir del conteo de sitios por sector, atribuyéndose que en cada sitio existe la presencia de una vivienda. En los diferentes sectores de la ciudad se consideró un total de 98 viviendas de un total de 371, según muestreo aleatorio estratificado. Entre estas viviendas encuestadas se encontraban casas de madera y de material sólido, de diferentes años de construcción. En todos los sectores la edad de las viviendas superó los 30 años. Además, se realizó una prospección en tres barracas o depósitos de madera, para determinar eventuales ataques de insectos xilófagos.

Para determinar la magnitud del daño se realizó una encuesta descriptiva en cada una de las viviendas, elegidas al azar, que consideró los siguientes antecedentes: año de construcción; estimación de la superficie total construida en metros cuadrados; frecuencia de daño (presencia o ausencia); tipo de madera atacada; grado de elaboración de la misma; porcentaje de daño según superficie de la vivienda y los síntomas de ataque. El cálculo del daño se basó en la visualización de los signos externos que se presentaron. Esta información fue resultado de entrevistas con los dueños de casa respectivos, con los cuales se clasificó la vivienda de acuerdo al porcentaje de daño según superficie, determinándose las diferentes categorías para cada una de ellas. Según estas categorías, se asignaron los siguientes niveles de deterioro: mínimo (1 a 25\%), moderado (26 a 50\%), severo (51 a $75 \%$ ) y muy severo (76 a 100\%). En los depósitos de madera se tomó en cuenta principalmente: el volumen producido al año, la frecuencia del daño, tipo o especie de madera, grado de elaboración, porcentaje de daño según volumen y los signos más comunes encontrados.

Para determinar las especies de coleópteros el trabajo de laboratorio se efectuó en dependencias de la Universidad de La Frontera, en el Instituto del Medio Ambiente y en la sección de Entomología de la Facultad de Ciencias Agropecuarias y Forestales. En laboratorio se mantuvieron las piezas de madera con síntomas de daño en cámara bioclimática, bajo condiciones ambientales controladas $\left(15^{\circ} \mathrm{C}\right.$ y 9 horas de luz), para obtener las especies causantes del daño.

Debido a las variables registradas en este estudio, el análisis de datos consistió en contrastes de asociación utilizando tablas de contingencia, para evaluar la dependencia entre variables categóricas, a través del valor crítico de la prueba chi-cuadrado de Pearson, empleando un nivel de significancia del 5\%. La dependencia consideró la relación entre las siguientes variables: año de construcción de las viviendas y frecuencia de daño, año de construcción y categorías de daño, según superficie, tipo de madera atacada y categorías de daño, signos externos de ataque y categorías de daño y por último sectores muestreados versus categorías de daño, según superficie.

\section{RESULTADOS}

De las 98 viviendas muestreadas en los diferentes sectores urbanos descritos para la comuna de Padre las Casas, un total de 71 viviendas presentó daños por coleópteros xilófagos (equivalente a un 70\%), siendo el sector Villa los Canelos el más afectado con un $89 \%$. Las viviendas que presentaron estructuras de madera nativa y una combinación de éstas con madera exótica evidenciaron un 2 y $14 \%$ de daño, respectivamente. El grado de elaboración de la madera de construcción que manifestó mayor daño correspondió a la madera pintada con un $44 \%$. Sin embargo, la mayoría de las viviendas presentaba protecciones de pintura antigua, lo que explica este resultado. Las maderas en bruto y en bruto y pintada, según categoría, presentan bajos síntomas de daño, debido probablemente a la mayor preocupación de los pobladores de remodelar y cambiar piezas de maderas dañadas o simplemente al menor uso que llevaban éstas en servicio.

Año de construcción y categorías de daño. La dependencia que resultó entre la variable influyente, año de construcción, sobre la variable respuesta, categorías de daño, fue estadísticamente significativa. En la figura 1 se muestra que las viviendas construidas entre los años 1965 a 1971 concentran el mayor daño, aproximadamente un $81 \%$. Cabe destacar que las viviendas construidas a partir del año 1993, no presentaron síntomas de deterioro (figura 1). Un $42 \%$ de las viviendas presentó niveles de dete- 
rioro moderado y un $38 \%$ un nivel severo, según el daño en su superficie construida.

Tipo de madera atacada y categorías de daño. No se estableció asociación entre el tipo de madera atacada y las respectivas categorías de daño. Sin embargo, los cuatro niveles de deterioro se concentran en la madera exótica, existiendo en este tipo una mayor frecuencia de daño (figura 2).

Signos externos de ataque y categorías de daño. No se encontró asociación entre estas variables. Sin embargo, al igual que el caso anterior, se aprecia en la figura 3 una concentración de los mayores niveles de deterioro en forma de agujeros y aserrín.

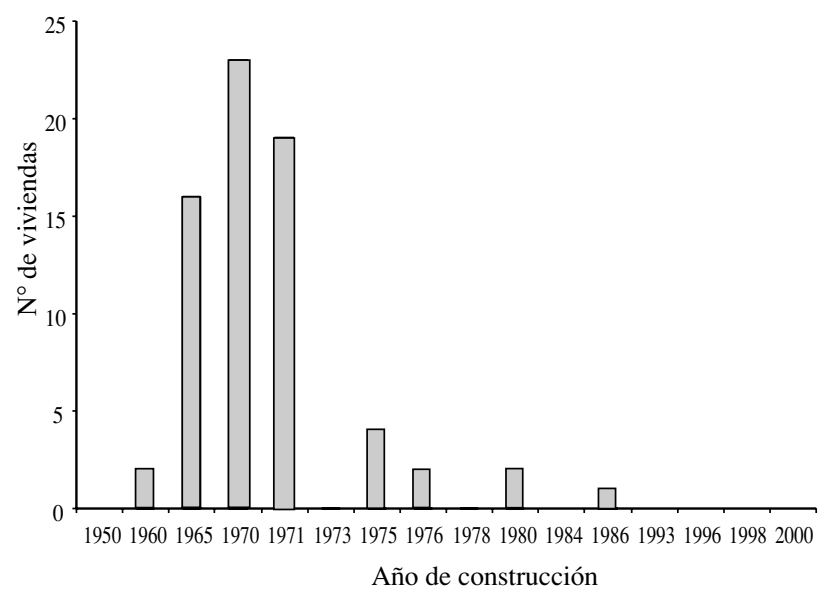

Figura 1. Síntomas de daño causados por coleópteros xilófagos y edad de las viviendas en Padre Las Casas (La Araucanía, Chile).

Damage symptoms by xylophagous Coleopteran and house age in Padre Las Casas (La Araucanía, Chile).

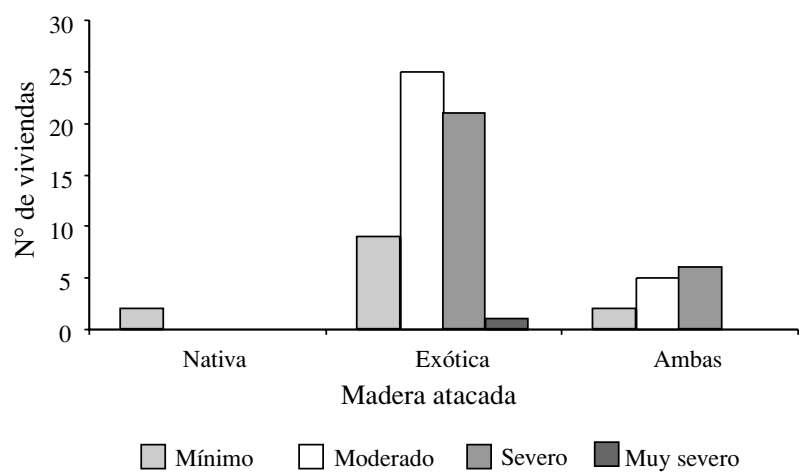

Figura 2. Origen de la madera de construcción y niveles de deterioro en viviendas de Padre Las Casas (La Araucanía, Chile).

Wood origin and house deterioration in Padre Las Casas (La Araucanía, Chile)

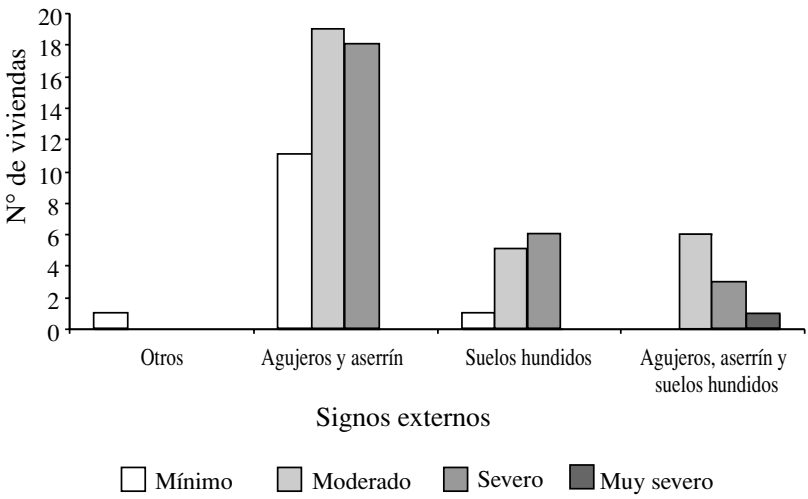

Figura 3. Síntomas externos de deterioro de viviendas en Padre Las Casas (La Araucanía, Chile).

External symptoms of house deterioration in Padre Las Casas (La Araucanía, Chile)

Especies de coleópteros xilófagos encontrados. Las tres especies de coleópteros relacionados con daños en madera fueron determinadas como los Anobiidae Anobium punctatum (De Gueer), Hadrobregmus punctatipennis Pic y el Curculionidae Pentarthrum huttoni Wollaston. Las observaciones de laboratorio indicaron que A. punctatum presentó un ciclo de vida monovoltino. Para el caso de $P$. huttoni, sólo se obtuvieron registros durante las observaciones obtenidas en terreno, sin poder determinar su desarrollo en laboratorio. El número total de individuos encontrados fue cercano a un centenar, correspondiendo 55 ejemplares a $P$. huttoni, 40 a $A$. punctatum y el resto a $H$. punctatipennis

\section{DISCUSIÓN}

Los síntomas de daño se presentaron principalmente en la madera estructural, siendo la madera exótica la más atacada con un 54\%. En estas viviendas se destaca el uso muy frecuente de pino insigne, especie muy susceptible al ataque de coleópteros xilófagos, coincidiendo estos valores con los citados por Peters et al. (1996) y Rodríguez (1998), especialmente para insectos de la familia Anobiidae, particularmente A. punctatum.

La relación de año de construcción y categorías de daño (figura 1) concuerda con la antigüedad de las construcciones. Al respecto, varios autores como Hadlington (1996), Peters et al. (1996) y Rojas (2000), señalan que el daño ocasionado por insectos xilófagos ocurre en maderas con más de 20 años de servicio. Distinta apreciación se observó en las viviendas de la Comuna de Padre Las Casas, cuya construcción es más reciente, en las que sólo algunas presentaron un nivel de deterioro de grado moderado (figura 1). 
De acuerdo al tipo de madera atacada y categorías de daño (figura 2), el no encontrarse en este trabajo una asociación entre la madera infestada y categorías de daño, en los cuales el mayor nivel de deterioro lo presentaron las maderas exóticas puede explicarse, según Rodríguez (1998), a que el tipo de madera atacada por coleópteros xilófagos se relaciona con maderas de latifoliadas y coníferas extranjeras. Tratándose de especies exóticas, es probable que los coleópteros xilófagos prefieran las maderas no nativas.

Con respecto a los signos externos de ataque y categorías de daños (figura 3), el no encontrarse una asociación entre estas variables pero sí una abundante presencia de aserrín y agujeros de salida de estos insectos, concuerda con Mourier et al. (1979), quienes consideran que los agujeros encontrados en la madera son frecuentemente la prueba de los graves ataques de estos escarabajos. Igualmente Rodríguez (1998) menciona que la determinación de los daños se debe a la presencia de galerías larvarias circulares con aserrín de cierta granulometría.

Finalmente, de los insectos registrados en el estudio, no fue recolectada la especie Lyctus chilensis Gerber (Lyctidae), la que según Vargas (2002), Luppichini y Ripa (2004) y Rojas y Gallardo (2004) se encuentra ampliamente distribuida en el país.

\section{CONCLUSIONES}

Las especies de coleópteros xilófagos asociados con madera en servicio correspondieron a los Anobiidae Anobium punctatum (De Gueer), Hadrobregmus punctatipennis Pic y al Curculionidae Pentarthrum huttoni Wollaston. La primera y última especie son importantes y su control es necesario para detener la infestación a nuevas viviendas de madera. No se detectó la presencia de $L y c$ tus chilensis ni la termita subterránea.

En los cuatro sectores evaluados se concluye que el daño es considerable, pues el $70 \%$ de las viviendas mostró síntomas de deterioro por estos insectos. El sector más afectado corresponde a la Villa Los Canelos, con un $89 \%$, debido a la mayor antigüedad del sector, poca durabilidad natural de las maderas empleadas en la construcción de dichas viviendas y los mínimos cuidados que le dan sus pobladores. La madera exótica es la más perjudicada, con un 54\%. En éstas se destaca el uso muy frecuente de pino insigne, el cual constituye una especie muy susceptible al ataque de coleópteros xilófagos.

La frecuencia de daño presentada en los diferentes sectores mostró diferencias significativas, con respecto al año de construcción de las viviendas, concentrándose el daño principalmente en las casas más antiguas, con un $82 \%$ de representatividad.

Las categorías de daño más frecuentes encontrados en las viviendas correspondieron a los niveles moderado y severo, con un 42 y $38 \%$, respectivamente, mostrando diferencias significativas y coincidiendo con la mayor antigüedad en sus construcciones.

\section{AGRADECIMIENTOS}

Los autores agradecen al Programa de Aplicación Sistema de Financiamiento tesis Pro Desarrollo IX Región, financiado por FNDR IX Región de La Araucanía.

\section{REFERENCIAS}

Apablaza J. 2000. Introducción a la entomología general y agrícola. Textos universitarios. Facultad de Agronomía e Ingeniería Forestal. Santiago, Chile. Ediciones Universidad Católica de Chile. 339 p.

Artigas J. 1994. Entomología económica. Insectos de interés agrícola, forestal, médico veterinario (Nativos, introducidos y susceptibles de ser introducidos). Vol. 2. Concepción, Chile. Universidad de Concepción. 943 p.

Barriga J, S Curkovic, T Fichet, J Henríquez, J Macaya. 1993. Nuevos antecedentes de coleópteros xilófagos y plantas hospederas en Chile, con una recopilación de citas previas. Rev. Chilena de Ent. 20:65-91.

Creffield JW. 1996. Wood destroying insects, wood borers and termites. Second edition. Australia. CSIRO. 44 p.

Coulson R, J Writter. 1990. Entomología Forestal Ecología y Control. México D.F., México. Limusa. 751 p.

Dajoz R. 2001. Entomología Forestal. Los insectos y el bosque. Madrid, España. Mundi Prensa. 549 p.

Hadlington P. 1999. Australian termites and other common timber pest. Second edition. Australia. UNSW Press. $126 \mathrm{p}$.

I.G.M (Instituto Geográfico Militar). 1993. Atlas Geográfico de Chile. Tercera edición. Santiago, Chile. Instituto Geográfico Militar. 139 p.

Klein C, D F Waterhouse. 2000. Distribución e importancia de los artrópodos asociados a la agricultura y silvicultura en Chile. Monograph $\mathrm{N}^{\circ}$ 68. Canberra, Australia. ACIAR. $231 \mathrm{p}$.

Luppichini P, R Ripa. 2004. Coleópteros xilófagos presentes en la madera en Chile. In Ripa y Luppichini (eds.). 2004. Termitas y otros insectos xilófagos en Chile: Especies, biología y manejo. Santiago, Chile. Colección Libros INIA № 11. p. 19-40.

Mourier H, O Winding, E Sunesen. 1979. Guía de los animales parásitos de nuestras casas. Barcelona, España. Omega. $224 \mathrm{p}$.

Peters Bc, J King, Fr Wylie. 1996. Pests of Timber in Queensland. Queensland Forestry Research Institute, Department of Primary Industries, Brisbane, 175 p. Consultado 19 febrero 2002. Disponible en: http://www.rlq.dcilgp.qld. gov.au/home/default.html

Prado E. 1991. Artrópodos y sus enemigos naturales asociados a plantas cultivadas en Chile. Serie Boletín Técnico 169. Santiago, Chile. Instituto de Investigaciones Agropecuarias. 203 p. 
Rodríguez J. 1998. Patología de la madera. Escuela técnica superior de Ingenieros de Montes. Fundación Conde del Valle de Salazar. España. Ediciones Mundi-Prensa. 349 p.

Rojas E. 2000. Insectos asociados a madera en Chile. Documento interno, laboratorio regional SAG-Osorno, Chile. $14 \mathrm{p}$.

Rojas E, R Gallardo. 2004. Manual de insectos asociados a maderas en la zona sur de Chile. División Protección Agrícola. Proyecto Vigilancia y Control de Plagas Forestales. Santiago, Chile. Servicio Agrícola y Ganadero. 64 p.
Torres J. 1968. Los organismos xilófagos y su tratamiento. Madrid, España. Ministerio de Agricultura. Dirección de Montes, Caza y Pesca Fluvial. 51 p.

Vargas S. 2002. Insectos xilófagos asociados a madera en servicio en inmuebles de la ciudad de Valdivia, X Región. Tesis Ingeniero Forestal. Valdivia, Chile. Facultad de Ciencias Forestales. Universidad Austral de Chile. 44 p.

Yela J. 1997. Insectos causantes de daños al patrimonio histórico y cultural: caracterización, tipos de daños y métodos de lucha (Arthropoda: Insecta). Bol. Sociedad Entom. Aragonesa 20:111-122.

Recibido: 16.08 .04

Aceptado: 09.03.06 